\title{
Intestinal obstruction due to primary intestinal melanoma in a patient with a history of rectal cancer resectioning: A case report
}

\author{
GANG LI* , XIAOJIANG TANG* , JIANJUN HE and HONG REN \\ Department of Surgical Oncology, The First Affiliated Hospital of School of Medicine, \\ Xi'an Jiaotong University, Xi'an, Shaanxi 710061, P.R. China \\ Received February 18, 2013; Accepted September 9, 2013
}

DOI: $10.3892 / \operatorname{mco} .2013 .217$

\begin{abstract}
The vast majority of the cases of intestinal melanomas are metastatic lesions, originating from an occult primary cutaneous or ocular lesion, whereas primary small intestinal melanomas are extremely rare. This is a rare case of primary small intestinal malignant melanoma with intestinal obstruction in a patient with a prior history of rectal cancer resection. The patient was admitted for abdominal pain and obstipation. Following an overall inspection, the patient was subjected to surgical treatment and a small intestinal tumor was removed. The histopathological examination of the lesion revealed a diffuse neoplastic infiltration involving the entire thickness of the intestinal mucosa. The neoplastic cells exhibited marked atypia, pleomorphism and immunoreactivity to S-100, anti-melanoma antibody (HMB-45) and melanocyte/melanoma tumor antigen (Melan-A). The diagnosis of primary small intestinal melanoma was confirmed. The patient underwent an uneventful postoperative recovery and was administered adjuvant therapy. At the 3-month, 6-month and 1-year follow-up, the patient remained alive, with no signs of tumor metastasis and/or recurrence. In this case, the patient was repetitively assessed by abdominal computed tomography (CT) and plain film, confirming that the obstruction was caused by small intestinal melanoma. There was no association between the rectal cancer history and the melanoma. A definitive diagnosis requires detailed clinical, histopathological and immunohistochemical analyses.
\end{abstract}

Correspondence to: Professor Jianjun He, Department of Surgical Oncology, The First Affiliated Hospital of School of Medicine, Xi'an Jiaotong University, 117 Yanta Road, Xi'an, Shaanxi 710061, P.R. China

E-mail: dooopenit@163.com

*Contributed equally

Key words: intestinal obstruction, primary small intestinal melanoma, rectal cancer

\section{Introduction}

Primary melanoma originating in the small intestine is extremely rare. It is commonly believed that the vast majority of cases are metastatic, originating from an occult primary cutaneous or ocular lesion. The diagnostic criteria for primary intestinal malignant melanoma have been determined. However, there is significant dispute over the treatment of primary intestinal malignant melanoma, since this type of tumor is associated with a very poor prognosis. There are usually no clinical manifestations in the early stages of this tumor; therefore, diagnosis is often delayed until the emergence of complications (1). This is the case report of a primary small intestinal malignant melanoma, complicated by intestinal obstruction, in a patient with a history of rectal cancer resection. Furthermore, the relevant literature was reviewed in order to improve our understanding of primary intestinal malignant melanoma.

\section{Case report}

A 65-year-old man was admitted to the Emergency Department of a local hospital, complaining of persistent abdominal pain and obstipation. Abdominal computed tomography (CT) revealed incomplete intestinal obstruction and the patient underwent fasting, gastrointestinal decompression, parenteral nutrition and inhibition of gastric acid secretion. However, there was no significant improvement in the symptoms.

Since the administered treatment was ineffective, the patient was admitted to our hospital. We found that the patient had undergone Miles' colorectal cancer resection 2 years earlier, followed by adjuvant chemotherapy (FOLFOX4) and regular inspection. However, there were no signs of recurrence and/or metastasis. Significant findings were limited to the abdomen, which was mildly tender to palpation in the periumbilical region. There was no tenderness or rebound phenomenon, the bowel sounds were hyperactive and there was no palpable mass. The rectal examination was normal. The admission laboratory values were normal, except for the low levels of albumin and potassium ion concentration. Following admission, the re-examination of the abdominal plain film indicated complete small intestinal obstruction (Fig. 1A) and 


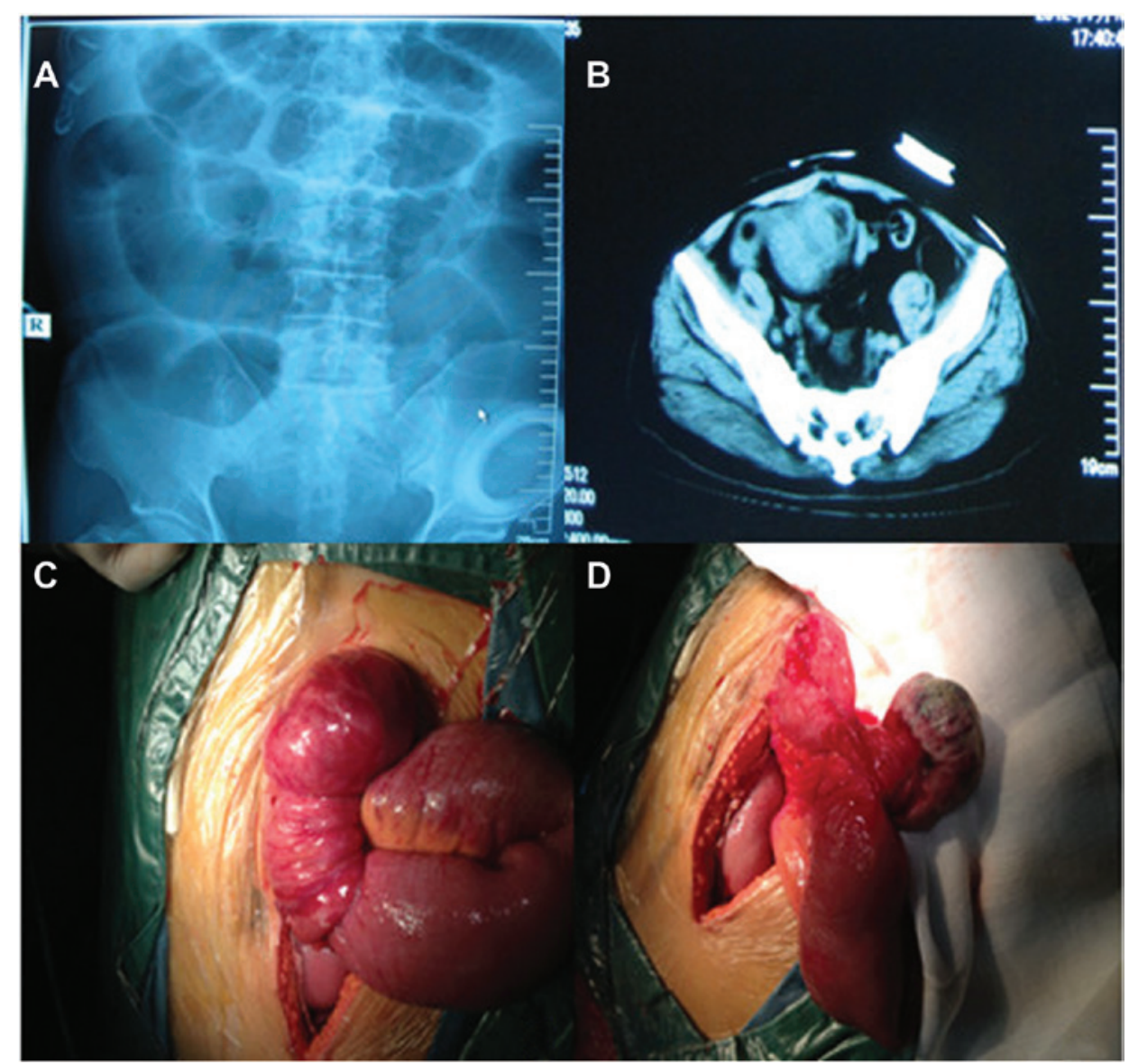

Figure 1. Patient imaging data and surgical procedure. (A) Small intestinal expansion and pneumatosis on abdominal plain film. (B) Computed tomography imaging of intestinal mass lesion in dilated ileal loop segment. (C) Invagination of the ileum. (D) Resected specimen showing a polypoid mass $(6 \mathrm{~cm}$ in diameter).

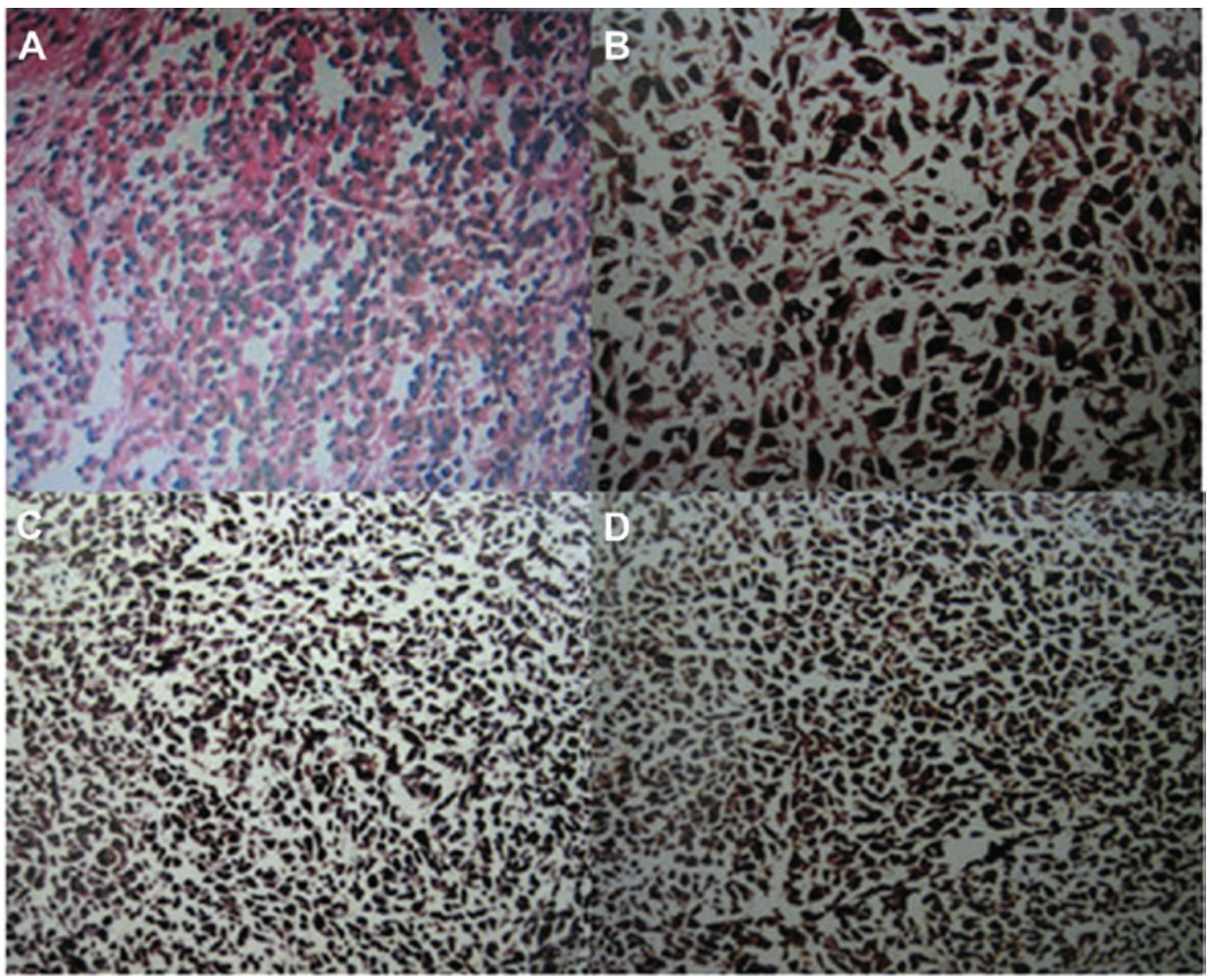

Figure 2. Histopathological characteristics of the malignant melanoma. (A) Loose arrangement of melanoma cells with melanin deposits (hematoxylin and eosin staining, magnification, x10). (B) Positivity for S-100 protein (immunostaining, magnification, x20). (C) Positivity for anti-melanoma antibody (immunostaining, magnification, x10). (D) Positivity for melanocyte/melanoma tumor antigen (immunostaining, magnification, x10). 
re-examination of the abdominal CT revealed small intestinal obstruction, expansion and pneumatosis, absence of the rectum and presence of a pelvic mass (Fig. 1B).

Abdominal ultrasonography revealed a distension of the intestinal loops, without additional signs of parenchymatous organ pathology. In order to exclude the recurrence of colon cancer a colonoscopy was performed and the findings were normal. Head and chest CT scan revealed no pathological changes. Therefore, an urgent surgical exploration was performed.

During laparotomy, a 6-cm mass was identified $15 \mathrm{~cm}$ from the distal end of the ileum. There was no other evidence of abdominal metastases and the liver was normal to palpation. The mass was pedunculated, leading to intestinal canal invagination and intestinal obstruction. The intestinal obstruction was relieved by surgical resection of the mass, including a segment of the ileum and the corresponding mesenterium (Fig. 1C and D). The pathological examination of the resected specimen confirmed the diagnosis of a small intestinal malignant melanoma, with invasion of the small intestinal muscle layer and absence of metastasis to the mesenteric lymph nodes (Fig. 2A). The immunohistochemical invetigation confirmed that the tumor cells were positive for S-100, anti-melanoma antibody (HMB-45) and melanocyte/melanoma tumor antigen (Melan-A) (Fig. 2B-D). The postoperative course was uneventful and the patient was discharged after 10 days. He was administered adjuvant chemotherapy and high-dose interferon (IFN) therapy. At the 3-month, 6-month and 1-year follow-up, the patient remained alive, with no signs of skin melanoma and/or tumor recurrence.

\section{Discussion}

Primary small intestinal melanoma is extremely rare. In a large series investigating 84,836 cases of malignant melanoma, only $1.3 \%$ originated from the gastrointestinal mucosa and the relevant literature is sparse (2). Malignant melanoma mainly occurs in the skin, eye, vulva, occasionally in the rectum and anus, the genital and upper gastrointestinal tracts, paranasal sinuses and the parotid gland.

It has been demonstrated (3) that cutaneous malignant melanoma originates from epidermal melanocytes or dermal nevus cells, both of which are derived from the neural crest. Race, genetic factors, trauma, irritation, viral infections, sun exposure and immune disturbances may contribute to its progression. The origin of the mucous membrane malignant melanoma has not been elucidated. It was previously reported that the reason for the occurrence of malignant melanoma in non-exposed areas may be the effect of the sunlight on the skin, leading to the release of a substance into the circulation, thus affecting the melanocytes of the non-exposed skin areas or the mucous membranes (3). It was also hypothesized that radiation and growth factors lead to melanocyte hyperplasia and the subsequent development of malignant melanoma (4). The etiology of primary intestinal melanomas has not been determined. The proposed primary sources are the melanoblasts of the neural crest or the amine precursor uptake and decarboxylation (APUD) cells, which migrate to the ileum through the omphalomesenteric canal and undergo neoplastic transformation in non-cutaneous sites (5). However, this theory may explain rectal melanoma (6). Malignant melanoma usually metastasizes via hematogenous and lymphatic routes to the liver, lung, bone and brain. Malignant melanomas of the digestive system are usually metastatic lesions. In one case series, melanoma commonly metastasized to the liver (68\%), small intestine (58\%), colon (22\%) and stomach (20\%) (7). Small intestinal malignant melanoma is usually asymptomatic. Tumor growth is often accompanied by gastrointestinal tract hemorrhage, intussusception, obstruction, abdominal pain, nausea, vomiting and weight loss. The time between primary melanoma diagnosis and the development of digestive system metastases varies between 2 and 180 months (8). In the case of small intestinal malignant melanoma, surgical resection is the first-line treatment option, due to acceptable mortality and morbidity rates and the beneficial effect over the course of the disease (9-11).

In this case, the patient had a history of rectal cancer resection, leading to the preoperative suspicion of rectal cancer recurrence as the cause of intestinal obstruction. During laparotomy, a small, pedunculated, highly mobile intestinal tumor was identified. Intussusception was the result of the movement of the tumor after reaching a certain size. The patient was eventually diagnosed with primary small intestinal malignant melanoma. We hypothesize that the immunosuppressive state associated with colorectal cancer may have induced the development of the primary small intestinal malignant melanoma postoperatively. However, there is not enough evidence to support this hypothesis (12). To determine whether the small intestinal malignant melanoma was a primary lesion, Sachs et al (13) established three diagnostic criteria: i) single lesion; ii) other organs free of primary lesions and absence of enlargement of the draining lymph nodes; and iii) survival time $>1$ year after diagnosis. In the present case, the patient was eventually diagnosed with intestinal obstruction caused by a tumor, according to the preoperative systemic CT and abdominal plain film, with no detection of metastatic lesions in other viscera. The postoperative pathological examination confirmed the diagnosis of primary small intestinal melanoma and there was no association between the colorectal cancer history and intestinal obstruction. The pathological examination also confirmed that there was no metastasis to the draining regional lymph nodes. The patient's skin, mucous membranes and eyes were thoroughly examined postoperatively and no pathological changes were identified. Furthermore, the survival period exceeded 1 year. Therefore, the patient was diagnosed with primary small intestinal malignant melanoma.

Melanomas arising on mucosal surfaces appear to be more aggressive and are associated with worse prognosis compared to cutaneous melanomas. The poorer prognosis may be associated with the delay in diagnosis, more aggressive behaviour, or earlier dissemination due to the rich lymphatic and vascular supply of the gastrointestinal mucosa (14). For isolated intestinal malignant melanoma, surgery may prolong survival (15). Previous studies demonstrated that postoperative adjuvant chemotherapy and IFN treatment may be beneficial for the patients, although their role is limited (16-18). Therefore, early diagnosis and surgical resection of the primary small intestinal malignant melanoma is crucial in improving the prognosis.

In conclusion, the patient presented with intestinal obstruction after undergoing resection of colorectal cancer and it 
was first considered that the reason was tumor recurrence. Therefore, a full preoperative evaluation was performed, which enabled timely diagnosis and treatment. Surgical resection and postoperative adjuvant therapy may significantly improve the prognosis of patients with primary small intestinal malignant melanoma. Therefore, a full preoperative assessment of cancer patients is recommended, possibly including positron emission tomography/CT, to ensure optimal surgical planning.

\section{References}

1. Jorge E, Harvey HA, Simmonds MA, Lipton A and Jaehl RJ: Symptomatic malignant melanoma of the gastrointestinal tract. Operative treatment and survival. Ann Surg 199: 328-331, 1984.

2. Chang AE, Karnell LH and Menck HR: The National Cancer Data Base report on cutaneous and noncutaneous melanoma: a summary of 84,836 cases from the past decade. The American College of Surgeons Commission on Cancer and the American Cancer Society. Cancer 83: 1664-1678, 1998.

3. Takubo K, Kanda Y, Ishii M, et al: Primary malignant melanoma of the esophagus. Hum Pathol 14: 727-730, 1983.

4. Capizzi PJ and Donohue JH: Metastatic melanoma of the gastrointestinal tract: a review of the literature. Compr Ther 20: 20-23, 1994.

5. Amar A, Jougon J, Edouard A, Laban P, Marry JP and Hillion G: Primary malignant melanoma of the small intestine. Gastroenterol Clin Biol 16: 365-367, 1992 (In French).

6. Clemmensen OJ and Fenger C: Melanocytes in the anal canal epithelium. Histopathology 18: 237-241, 1991.

7. Klausner JM, Skornick Y, Lelcuk S, Baratz M and Merhav A: Acute complications of metastatic melanoma to the gastrointestinal tract. Br J Surg 69: 195-196, 1982.
8. Arneja JS and Gosain AK: Giant congenital melanocytic nevi. Plast Reconstr Surg 120: 26e-40e, 2007.

9. Atmatzidis KS, Pavlidis TE, Papaziogas BT and Papaziogas TB Primary malignant melanoma of the small intestine: report of a case. Surg Today 32: 831-833, 2002.

10. Branum GD and Seigler HF: Role of surgical intervention in the management of intestinal metastases from malignant melanoma. Am J Surg 162: 428-431, 1991.

11. Butte JM, Meneses M, Waugh E, et al: Ileal intussusception secondary to small bowel metastases from melanoma. Am J Surg 198: e1-2, 2009.

12. Oosterling SJ, van der Bij GJ, Mels AK, Beelen RH, Meijer S, van Egmond $\mathrm{M}$ and van Leeuwen PA: Perioperative IFN-alpha to avoid surgically induced immune suppression in colorectal cancer patients. Histol Histopathol 21: 753-760, 2006.

13. Sachs DL, Lowe L, Chang AE, et al: Do primary small intestinal melanomas exist? Report of a case. J Am Acad Dermatol 41: 1042-1044, 1999.

14. Lagoudianakis EE, Genetzakis M, Tsekouras DK, Papadima A, Kafiri G, Toutouzas K, Katergiannakis V and Manouras A: Primary gastric melanoma: a case report. World J Gastroenterol 12: 4425-4427, 2006.

15. Sanki A, Scolyer RA and Thompson JF: Surgery for melanoma metastases of the gastrointestinal tract: indications and results. Eur J Surg Oncol 35: 313-319, 2009.

16. Ollila DW, Essner R, Wanek LA and Morton DL: Surgical resection for melanoma metastatic to the gastrointestinal tract. Arch Surg 131: 975-980, 1996.

17. Kadivar TF, Vanek VW and Krishnan EU: Primary malignant melanoma of the small bowel: a case study. Am Surg 58: 418-422, 1992.

18. Letovanec I, Vionnet M and Bouzourene H: Primary appendiceal melanoma: fiction or reality? Hum Pathol 35: 627-629, 2004. 